\title{
Therapie der Cheilitis granulomatosa mit Fumarsäureestern: Ergebnisse einer prospektiven nichtkontrollierten Studie
}

\author{
Treatment of Cheilitis Granulomatosa with Fumaric Acid Esters: Results of a Prospective Noncontrolled Study
}

Autoren

Institut

\section{T. Jansen, S. Grabbe}

Klinik und Poliklinik für Dermatologie, Venerologie und Allergologie der Universität Essen (Direktor: Prof. Dr. Stephan Grabbe)
Bibliografie

Dol $10.1055 / \mathrm{s}-2007-966225$

Akt Dermatol 2007; 33:

72-75 (c) Georg Thieme

Verlag KG Stuttgart · New York ISSN 0340-2541

Korrespondenzadresse

Dr. Thomas Jansen

Klinik und Poliklinik für Dermatologie, Venerologie und Allergologie der Universität Essen

Hufelandstr. 55, 45122 Essen thomas.jansen@medizin.uniessen.de

\section{Zusammenfassung \\ $\nabla$}

Die Cheilitis granulomatosa bezeichnet eine seltene chronische Lippenschwellung auf dem Boden einer granulomatösen Entzündung. Berichtet wird über 5 Patienten im Alter von 25-56 (durchschnittlich 35,2) Jahren mit seit 5-18 (durchschnittlich 11,6) Monaten bestehender typischer Cheilitis granulomatosa. Bei 3 Patienten war zusätzlich eine Lingua plicata vorhanden. Bei allen Patienten war mindestens ein erfolgloser Therapieversuch vorangegangen. Es erfolgte eine orale Therapie mit Fumarsäureestern über insgesamt 5-6 (durchschnittlich 5,8) Monate. Eine deutliche Befundbesserung mit Reduzie-

\section{Einleitung}

$\nabla$

Die von Miescher [17] 1945 beschriebene Cheilitis granulomatosa ist eine seltene persistierende Lippenschwellung (Makrocheilie) auf dem Boden einer granulomatösen Entzündung unbekannter Ursache. Sie ist Teilsymptom des Melkersson-Rosenthal-Syndroms, das in klassischer Ausprägung zusätzlich Lingua plicata und Fazialisparese umfasst. Cheilitis granulomatosa und Melkersson-Rosenthal-Syndrom werden dem Spektrum der orofazialen Granulomatose zugeordnet [24]. Der Lippenschwellung entsprechen histologisch Lymphangiektasien, lymphohistiozytäre Infiltrate sowie nichtverkäsende epitheloidzellige Granulome. Die Erkrankung nimmt einen chronischen Verlauf mit selten zu beobachtender spontaner Remission. Betroffen sind überwiegend junge Erwachsene ohne erkennbare Geschlechtspräferenz. Eine allgemein wirksame Therapie der Cheilitis granulomatosa ist derzeit nicht verfügbar.

Aufbauend auf die ermutigenden Ergebnisse der oralen Behandlung anderer granulomatöser Dermatosen wie kutane Sarkoidose $[2,7,21]$, anulä- rung der Lippenschwellung trat bei 3 Patienten ein, während die Lippenschwellung bei weiteren 2 Patienten vollständig abheilte. Innerhalb einer Nachbeobachtungsperiode von 4-8 (durchschnittlich 5,6) Monaten trat kein Rezidiv auf. Die Lingua plicata blieb durch die Therapie unbeeinflusst. Keine der überwiegend in der Initialphase der Therapie beobachteten Nebenwirkungen veranlassten zum vorzeitigen Therapieabbruch. Angesichts der begrenzten Therapiemöglichkeiten bei der Cheilitis granulomatosa weisen die erzielten Ergebnisse mit Fumarsäureestern auf einen möglichen Nutzen der Substanzen bei dieser Indikation hin, jedoch sind weitere kontrollierte Studien erforderlich.

res elastolytisches Riesenzellgranulom [2,6], Granuloma anulare disseminatum $[2,11,26]$ und Necrobiosis lipoidica $[2,4,12]$ mit Fumarsäureestern wurde dieses Therapiekonzept bei 5 Patienten mit therapierefraktärer Cheilitis granulomatosa verfolgt.

\section{Charakterisierung des Patienten- kollektivs, Therapie und Verlauf $\nabla$}

Berichtet wird über 5 Patienten (3 Männer, 2 Frauen) im Alter von 25-56 (durchschnittlich 35,2 ) Jahren mit Cheilitis granulomatosa, die in der Ambulanz der Universitäts-Hautklinik Essen rekrutiert wurden ( $\bullet$ Tab. 1 ).

Bei allen Patienten kam es zu einer schubweisen, später zu persistierender, diffuser, entzündlicher Lippenschwellung, die zum Zeitpunkt der ambulanten Vorstellung seit 5-18 (durchschnittlich 11,6) Monaten bestand ( $\bullet$ Abb. 1 a). Temperaturerhöhung oder Krankheitsgefühl in Begleitung der Schübe war bei keinem der Patienten eruierbar. Bei allen Patienten war die Schwellung auf die Lippen beschränkt, wobei bei jeweils $2 \mathrm{~Pa}$ - 
Tab. 1 Kenndaten der Patienten mit Cheilitis granulomatosa, die mit Fumarsäureestern behandelt wurden

\begin{tabular}{|c|c|c|c|c|c|c|}
\hline Pat.-Nr. & $\begin{array}{l}\text { Alter (Jahre), } \\
\text { Geschlecht }\end{array}$ & Lokalisation & $\begin{array}{l}\text { Vorbehandlung, Dosie- } \\
\text { rung, Dauer (Monate) }\end{array}$ & $\begin{array}{l}\text { Bestandsdauer } \\
\text { (Monate) }\end{array}$ & $\begin{array}{l}\text { Behandlungsdauer } \\
\text { (Monate), Ergebnis }\end{array}$ & $\begin{array}{l}\text { Nachbeobachtung } \\
\text { (Monate), Rezidiv }\end{array}$ \\
\hline 1 & $33, \mathrm{~m}$ & Oberlippe* & $\begin{array}{l}\text { Clofazimin, } 100 \text { mg/die, } 2 \\
\text { Ketotifen, } 1 \text { mg/die, } 3\end{array}$ & 18 & $\begin{array}{l}\text { 6, deutliche } \\
\text { Besserung }\end{array}$ & 4 , nein \\
\hline 2 & $25, w$ & Unterlippe* & $\begin{array}{l}\text { Triamzinolonazetonid } \\
\text { intraläsional, } \\
5 \mathrm{mg} / \text { Monat, } 2\end{array}$ & 5 & 5, Abheilung & 8 , nein \\
\hline 3 & $56, \mathrm{~m}$ & Unterlippe & Clofazimin, $100 \mathrm{mg} / \mathrm{die}, 5$ & 12 & 6, Abheilung & 5 , nein \\
\hline 4 & $26, \mathrm{~m}$ & Oberlippe & $\begin{array}{l}\text { Triamzinolonazetonid } \\
\text { intraläsional, } \\
5 \text { mg/Monat, } 3\end{array}$ & 8 & $\begin{array}{l}6 \text {, deutliche } \\
\text { Besserung }\end{array}$ & 6 , nein \\
\hline 5 & $36, w$ & $\begin{array}{l}\text { Ober- und } \\
\text { Unterlippe }^{*}\end{array}$ & $\begin{array}{l}\text { Clofazimin, } 100 \text { mg/die, } 4 \\
\text { Prednisolon, } 1,0 \mathrm{mg} / \mathrm{kg} \\
\text { KG/die, } 1\end{array}$ & 15 & $\begin{array}{l}6 \text {, deutliche } \\
\text { Besserung }\end{array}$ & 5 , nein \\
\hline
\end{tabular}

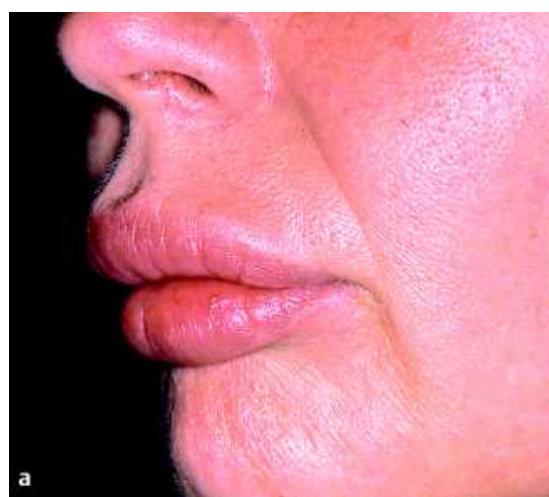

Abb. 1 36-jährige Patientin (Pat.-Nr. 5) mit Cheilitis granulomatosa der Ober- und Unterlippe (a) sowie Lingua plicata (b).

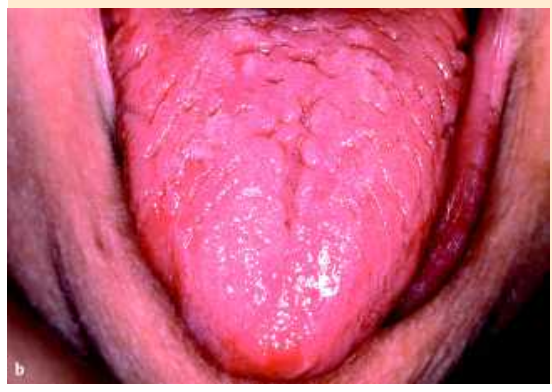

tienten die Ober- oder Unterlippe und bei 1 Patienten gleichzeitig die Ober- und Unterlippe betroffen waren. Palpatorisch war eine vermehrte Konsistenz ohne Druckschmerzhaftigkeit zu vermerken. Die Oberfläche der Lippen war normalfarben und wies bei 2 Patienten Rhagaden auf. Subjektive Beschwerden in Form von Spannungsgefühl und Parästhesien wurden von 4 Patienten angegeben.

Bei 3 Patienten war zusätzlich eine verstärkte Furchung der vorderen zwei Drittel der Zungenoberfläche im Sinne einer Lingua plicata, jedoch ohne Geschmacksstörungen, vorhanden ( Abb. 1 b). Dagegen waren Wangen, Augenlider, Stirn, Gaumen oder Gingiva frei von gleichartigen Veränderungen.

Die regionalen Lymphknoten waren weder vergrößert noch druckschmerzhaft tastbar.

Begleiterkrankungen waren nicht eruierbar. Medikamente wurden nicht eingenommen. Hinweise auf Herpes simplex recidivans oder stattgehabtes Gesichtserysipel ergaben sich anamnestisch bei keinem Patienten. Gastrointestinale Beschwerden wurden nicht angegeben. In der Familienanamnese fand sich kein Anhalt für vergleichbare Hautveränderungen oder andere Teilsymptome des Melkersson-Rosenthal-Syndroms.
Mindestens eine erfolglose Vorbehandlung mit Glukokortikoiden oral oder intraläsional, Clofazimin oder Ketotifen war der ambulanten Vorstellung aller Patienten vorangegangen. In den letzten 4 Wochen hatte es keinen weiteren Therapieversuch gegeben.

Bei allen Patienten wurden zur Diagnosesicherung tiefe Stanzbiopsien von den betroffenen Lippen entnommen. Histologisch imponierten in wechselnder Ausprägung, jedoch einheitlich ein deutliches Ödem mit erweiterten Lymphgefäßen, interstitiell und perivaskulär gelegene lymphohistiozytäre Infiltrate mit Plasmazellen sowie nichtverkäsende epitheloidzellige Granulome. Mit Spezialfärbungen auf Pilze (PAS) und Bakterien (Gram, Ziehl-Neelsen) ließen sich keine auffälligen Befunde erheben, die polarisationsmikroskopische Untersuchung ergab keinen Hinweis auf Fremdkörper im Gewebe.

Die neurologische Untersuchung lieferte bei keinem Patienten Hinweise auf eine periphere Fazialisparese oder Beteiligung weiterer Hirnnerven oder auch Störungen des autonomen Nervensystems.

Die Röntgenaufnahme des Thorax in zwei Ebenen lieferte einen altersentsprechend unauffälligen Befund, insbesondere ohne Anhalt für eine hilär lokalisierte Lymphadenopathie.

Sämtliche im Rahmen der Ausschlussdiagnostik erhobenen Laborparameter waren negativ oder im Normbereich, darunter: C-reaktives Protein, C1-Esterase-Inhibitor-Konzentration und -Aktivität, Komplementfraktion C4, Angiotensin Converting Enzym, löslicher Interleukin-2-Rezeptor, T4/T8-Ratio, Gesamt-IgE, Antistreptolysintiter sowie Borrelien- und Lues-Serologie.

Allergologische Untersuchungen mit Pricktestung auf häufige Aero- und Nahrungsmittelallergene sowie Epikutantestung (Europäische Standardreihe) ergaben keine Hinweise auf klinisch relevante Soforttyp- oder Spättyp-Sensibilisierungen.

Bei allen Patienten wurde mit deren Einverständnis eine orale Behandlung mit Fumarsäureestern eingeleitet. Um ein optimales Wirkungs- und Verträglichkeitsprofil zu erreichen, erfolgte die Applikation einschleichend mit Dosissteigerung in Anlehnung an das bei der Psoriasis vulgaris empfohlene Therapieprotokoll bis auf 3 Tabletten Fumaderm ${ }^{\mathrm{R}}$ pro Tag [17]. Klinische und serologische Verlaufskontrollen wurden in vierwöchigen Abständen durchgeführt. Bei den beiden Patientinnen erfolgte vor Therapiebeginn ein Schwangerschaftsausschluss durch $\beta$-HCGBestimmung im Serum. Die Therapie wurde solange fortgeführt, bis eine deutliche Befundbesserung oder vollständige Abheilung erkennbar war. Daran schloss sich eine mehrmonatige Nachbeobachtungsperiode an. 
Die orale Therapie mit Fumarsäureestern erfolgte über insgesamt 5-6 (durchschnittlich 5,8) Monate. Eine deutliche Befundbesserung mit Reduzierung der Lippenschwellung trat bei 3 Patienten ein, während die Lippenschwellung bei weiteren $2 \mathrm{~Pa}-$ tienten vollständig abheilte. Eine rasche Reduzierung der Lippenschwellung innerhalb der ersten Behandlungswochen wurde in keinem Fall verzeichnet. Innerhalb eines Nachbeobachtungszeitraumes von 4-8 (durchschnittlich 5,6) Monaten wurde kein Rezidiv beobachtet. Die Lingua plicata blieb durch die Therapie unbeeinflusst. Von Kontrollbiopsien wurde mangels Einwilligung der Patienten abgesehen.

Die Therapie erwies sich als gut verträglich. Nebenwirkungen traten bei 3 Patienten überwiegend in der Initialphase der Therapie auf und bestanden in Flushreaktionen und gastrointestinalen Beschwerden (Magendruck, Völlegefühl, Diarrhö). Die häufigsten Laborveränderungen waren eine vorübergehende leichte Lymphozytopenie (10 - 25\% der Gesamtleukozytenzahl) und Eosinophilie (4-10\% der Gesamtleukozytenzahl). Keine der beobachteten Nebenwirkungen gab Anlaß zum vorzeitigen Therapieabbruch.

\section{Diskussion \\ $\nabla$}

Eine allgemein wirksame Therapie der Cheilitis granulomatosa gibt es bisher nicht. Als Therapie der Wahl gelten Glukokortikoide, die wegen ihrer antiinflammatorischen Eigenschaften oral und/oder intraläsional appliziert werden. Aufgrund der besseren Wirksamkeit wird die intraläsionale Injektion von Glukokortikoiden vielfach bevorzugt, wobei vor allem Triamzinolonazetonid zur Anwendung kommt [22]. Die hierbei verwendeten Konzentrationen liegen üblicherweise bei $10 \mathrm{mg} / \mathrm{ml}$, jedoch werden auch höhere Konzentrationen bis $40 \mathrm{mg} / \mathrm{ml}$ empfohlen [15]. Die Vorteile dieses Vorgehens liegen in einer Reduzierung des injizierten Volumens bei höherer Konzentration des Wirkstoffes und einer Verlängerung der Remissionsphase. Die häufigsten Nebenwirkungen der intraläsionalen Steroidinjektionen sind Hautatrophie und Pigmentstörungen. Die Therapie mit Glukokortikoiden ist durch den chronisch-rezidivierenden Verlauf der Erkrankung und die zu erwartenden Nebenwirkungen einer Langzeittherapie limitiert. Die Schwellung bildet sich zwar vielfach in kurzer Zeit zurück, jedoch ist nach Absetzen der Medikamente regelmäßig mit Rezidiven zu rechnen.

Als Therapiealternative wird das Lepramittel Clofazimin eingesetzt. Es ist in Deutschland nicht zugelassen und muss über die internationale Apotheke angefordert werden. Während es bei einer bereits persistierenden Lippenschwellung lediglich zu einer langsamen und unvollständigen Rückbildung führt [23], ist bei einem akuten Schub der Erkrankung bereits nach wenigen Tagen mit einer Rückbildung der Schwellung zu rechnen [20]. Trotz vereinzelt berichteter dauerhafter Abheilungen ist die Rezidivquote ähnlich hoch anzusetzen wie unter einer Therapie mit Glukokortikoiden. Der Wirkmechanismus von Clofazimin ist nicht bekannt, steht jedoch in möglichem Zusammenhang mit dem Phagozytose-stimulierenden Effekt dieser Substanz auf Makrophagen.

In Einzelfällen wurde über das erfolgreiche Ansprechen auf Hydroxychloroquin, Methotrexat, Sulfasalazin, Metronidazol, Minozyklin alleine oder in Kombination mit Prednisolon [27], Thalidomid [16], Tranilast [10], Dapson und Danazol berichtet $[28,29]$.
Bei chronisch-progredienter, nicht auf konventionelle Therapie ansprechender Lippenschwellung besteht die Möglichkeit einer chirurgischen Intervention in Form einer Lippenreduktionsplastik [13].

Aufbauend auf die ermutigenden Ergebnisse der Behandlung anderer granulomatöser Dermatosen wie kutane Sarkoidose $[2,7,21]$, anuläres elastolytisches Riesenzellgranulom [2,6], Granuloma anulare disseminatum $[2,11,26]$ und Necrobiosis lipoidica $[2,4,12]$ entschlossen wir uns, die Wirksamkeit von Fumarsäureestern bei 5 Patienten mit Cheilitis granulomatosa zu prüfen. Hierbei sprachen alle Patienten mit therapierefraktärer, erfolglos vorbehandelter Lippenschwellung nach insgesamt 5-6 (durchschnittlich 5,8) Monaten auf die Therapie mit Fumaderm ${ }^{\mathrm{R}}$ in einer Tagesdosis von bis zu 3 Tabletten an. Eine deutliche Befundbesserung mit Reduzierung der Lippenschwellung trat bei 3 Patienten und eine vollständige Abheilung bei weiteren $2 \mathrm{~Pa}-$ tienten ein, wobei innerhalb des Nachbeobachtungszeitraumes von 4-8 (durchschnittlich 5,6) Monaten kein Rezidiv beobachtet wurde. Die Therapie erwies sich über den gesamten Behandlungszeitraum als gut verträglich.

Es ist nicht sicher auszuschließen, dass die in der berichteten Patientengruppe erzielte Befundbesserung oder Abheilung auf eine spontane Rückbildung der Erkrankung zurückzuführen ist, zumal es einen Einzelbericht über die erfolglose mehrmonatige Behandlung der Lippenschwellung mit Fumarsäureestern in analoger Dosierung gibt [2]. Angesichts der Neigung zur Chronizität und geringen Spontanremissionsrate der Cheilitis granulomatosa ist ein therapeutischer Effekt der verabreichten Fumarsäureester jedoch als wahrscheinlich anzusehen.

Fumarsäureester sind Ester der Fumarsäure $\left(\mathrm{C}_{4} \mathrm{H}_{4} \mathrm{O}_{4}\right)$, einer ungesättigten, aliphatischen Dikarbonsäure. Sie stellen eine wirksame Therapieoption bei schweren Formen der Psoriasis dar. Während der Therapie wird eine kontinuierliche Suppression sowohl der T-Helfer- als auch der T-Suppressor-Lymphozyten und eine Stimulation der TH2-Immunantwort beobachtet $[8,18]$. Da die Psoriasis eine TH1-vermittelte entzündliche Dermatose darstellt, könnte die Modulation der übersteigerten entzündlichen TH1-Immunantwort (IL-2, IFN- $\gamma$ ) durch eine antientzündliche TH2-Immunantwort (IL-4, IL-5, IL-10) für den positiven Effekt auf die Erkrankung verantwortlich sein. Darüber hinaus könnte der antipsoriatische Effekt von Fumarsäureestern auf eine reduzierte Expression Antigen-präsentierender Monozyten und Makrophagen zurückzuführen sein [1].

Die Pathogenese der Cheilitis granulomatosa ist unbekannt. Favorisiert wird eine multifaktorielle Genese, zu der Infektionen (Fokalinfekte, Spirochäten, Mykobakterien etc.), Allergien (Nahrungsmittel und -zusatzstoffe etc.) und genetische Faktoren beitragen $[5,19]$. In den Läsionen wurde eine monoklonale Expansion von Lymphozyten nachgewiesen, die Folge einer chronischen Antigenstimulation sein könnte [3]. Die Zytokinproduktion durch diesen Lymphozytenklon könnte für die Granulombildung verantwortlich sein. Da sich aktivierte T-Helfer-Lymphozyten, die Interleukin-2-Rezeptoren exprimieren, in den Läsionen nachweisen ließen, liegt offenbar eine zellvermittelte Immunreaktion zugrunde [14,25]. Mit diesem pathogenetischen Konzept ist der histologische Befund bei der Cheilitis granulomatosa gut vereinbar. Zunächst findet sich eine ödematöse Schwellung, dann treten perivaskuläre, vorwiegend lymphozytäre Infiltrate hinzu, die einen wechselnden Anteil an Plasmazellen aufweisen. Erst später findet man die charakteristischen nichtverkäsenden epitheloidzelligen Granulome. 
Angesichts der begrenzten Therapiemöglichkeiten bei der Cheilitis granulomatosa weisen die erzielten Ergebnisse mit Fumarsäureestern auf einen möglichen Nutzen der Substanzen bei dieser Indikation hin. Künftige Plazebo-kontrollierte Studien an größeren Patientenkollektiven sind erforderlich, um diese Ergebnisse zu überprüfen.

\section{Abstract}

\section{Treatment of Cheilitis Granulomatosa with Fumaric Acid Esters: Results of a Prospective Noncontrolled Study \\ $\nabla$}

Cheilitis granulomatosa is a rare chronic swelling of one or both lips due to granulomatous inflammation. Five patients aged 25-56 (mean 35.2) years with typical cheilitis granulomatosa are reported. Lip swelling was present for 5-18 (mean 11.6) months. Lingua plicata was found in 3 patients. At least one uneffective therapeutic trial had been performed in each patient. All patients received oral treatment with fumaric acid esters for 5-6 (mean 5.8) months. After the treatment period, significant reduction of lip swelling was achieved in 3 patients, while clearing of skin lesions could be observed in 2 patients. There was no relapse within a follow-up period of 4-8 (mean 5.6) months. Lingua plicata remained unchanged. Side effects were most prominent during the initial treatment period, but did not lead to drop-outs. The results of this study suggest that fumaric acid esters may be a potentially beneficial therapeutic option for patients with cheilitis granulomatosa. However, further controlled trials are necessary to confirm these results.

\section{Literatur}

1 Asadullah A, Schmid H, Friedrich M, Randow F, Volk HD, Sterry W, Docke $W D$. Influence of monomethylfumarate on monocytic cytokine formation - explanation for adverse and therapeutic effects in psoriasis? Arch Dermatol Res 1997; 289: 623-630

2 Breuer K, Gutzmer R, Völker B, Kapp A, Werfel T. Therapy of noninfectious granulomatous skin diseases with fumaric acid esters. Br J Dermatol 2005; 152: 1290-1295

3 De Quatrebarbes J, Cordel N, Bravard P, Lenormand B, Joly P. Macrochéilite de Miescher et expansion lymphocytaire monoclonale. Deux cas. Ann Dermatol Venereol 2004; 131: 55-57

4 Gambichler T, Kreuter A, Freitag M, Pawlak FM, Brockmeyer NH, Altmeyer $P$. Clearance of necrobiosis lipoidica with fumaric acid esters. Dermatology 2003; 207: 422-424

5 Greene RM, Rogers RS $3^{\text {rd }}$. Melkersson-Rosenthal syndrome: a review of 36 patients. J Am Acad Dermatol 1989; 21: $1263-1270$

6 Gutzmer R, Breuer K, Kiehl P, Kapp A, Werfel T. Successful therapy of annular elastolytic giant cell granuloma with fumaric acid esters. Dermatology 2002; 205: 421 - 424

7 Gutzmer R, Kapp A, Werfel T. Erfolgreiche Therapie einer Haut- und Lungensarkoidose mit Fumarsäureestern. Hautarzt 2004; 55: 553 557

8 Höxtermann S, Nüchel C, Altmeyer P. Fumaric acid esters suppress peripheral CD4- and CD8-positive lymphocytes in psoriasis. Dermatology 1998; 196: $223-230$
9 Kano Y, Shiohara T, Yagita A, Nagashima M. Treatment of recalcitrant cheilitis granulomatosa with metronidazole. J Am Acad Dermatol 1992; 27: 629-630

10 Kato T, Tagami H. Successful treatment of cheilitis granulomatosa with tranilast. J Dermatol 1986; 13: 402 - 403

11 Kreuter A, Gambichler T, Altmeyer P, Brockmeyer NH. Treatment of disseminated granuloma annulare with fumaric acid esters. BMC Dermatology 2002; $2: 5$

12 Kreuter A, Knierim C, Stücker M, Pawlak FM, Rotterdam S, Altmeyer P, Gambichler T. Fumaric acid esters in necrobiosis lipoidica: results of a prospective noncontrolled study. Br J Dermatol 2005; 153: 802 - 807

13 Kruse-Lösler B, Presser D, Metze D, Joos U. Surgical treatment of persistent macrocheilia in patients with Melkersson-Rosenthal syndrome and cheilitis granulomatosa. Arch Dermatol 2005; 141: 1085-1091

14 Lim SH, Stephens P, Cao QX, Coleman S, Thomas DW. Molecular analysis of $T$ cell receptor beta variability in a patient with orofacial granulomatosis. Gut 1997; 40: 683-686

15 Medeiros M Jr, Araujo MI, Guimaraes NS, Freitas LA, Silva TM, Carvalho $E M$. Therapeutic response to thalidomide in Melkersson-Rosenthal syndrome: a case report. Ann Allergy Asthma Immunol 2002; 88: $421-424$

16 Miescher G. Über essentielle granulomatöse Makrocheilie (Cheilitis granulomatosa). Dermatologica 1945; 91: $57-85$

17 Mignogna MD, Fedele S, Russo LL, Adamo D, Satriano RA. Effectiveness of small-volume, intralesional, delayed-release triamcinolone injections in orofacial granulomatosis: a pilot study. J Am Acad Dermatol 2004; 51: 265-268

18 Mrowietz U, Christophers E, Altmeyer P et al. Treatment of severe psoriasis with fumaric acid esters: scientific background and guidelines for therapeutic use. Br J Dermatol 1999; 141: 424-429

19 Nagel F, Fölster-Holst R. Cheilitis granulomatosa. Melkersson-Rosenthal-Syndrom. Hautarzt 2006; 57: $121-126$

20 Neuhofer J, Fritsch P. Cheilitis granulomatosa (Melkersson-RosenthalSyndrom): Behandlung mit Clofazimin. Hautarzt 1984; 35: 459-463

21 Nowack U, Gambichler T, Hanefeld C, Kastner U, Altmeyer P. Successful treatment of recalcitrant cutaneous sarcoidosis with fumaric acid esters. BMC Dermatology 2002; 2: 15

22 Perez-Calderon R, Gonzalo-Garijo MA, Chaves A, de Argila D. Cheilitis granulomatosa of Melkersson-Rosenthal syndrome: treatment with intralesional corticosteroid injections. Allergol Immunopathol 2004; 32: $36-38$

23 Ridder GJ, Fradis M, Lohle E. Cheilitis granulomatosa Miescher: treatment with clofazimine and review of the literature. Ann Otol Rhinol Laryngol 2001; 110: 964 - 967

24 Rogers RS. Granulomatous cheilitis, Melkersson-Rosenthal syndrome, and orofacial granulomatosis. Arch Dermatol 2000; 136: 1557-1558

25 Ronnblom L, Forsum U, Evrin PE, Gillnas T, Nethander G. Intralesional T lymphocyte phenotypes and HLA-DR expression in Melkersson-Rosenthal syndrome. Int J Oral Maxillofac Surg 1986; 15: 614-619

26 Schulze-Dirks A, Petzoldt D. Granuloma anulare disseminatum - erfolgreiche Behandlung mit Fumarsäureester. Hautarzt 2001; 52: $228-230$

27 Stein SL, Mancini AJ. Melkersson-Rosenthal syndrome in childhood: successful management with combination steroid and minocycline therapy. J Am Acad Dermatol 1999; 41: 746 - 748

28 Sussman GL, Yang WH, Steinberg S. Melkersson-Rosenthal syndrome: clinical, pathologic, and therapeutic considerations. Ann Allergy 1992; 69: 187-194

29 van der Waal RI, Schulten EA, van der Meij EH, van de Scheur MR, Starink TM, van der Waal I. Cheilitis granulomatosa: overview of 13 patients with long-term follow-up - results of management. Int J Dermatol 1992; 41: 225 - 229 